\title{
Características de los casos con dislipidemias mixtas en un estudio de población: resultados de la Encuesta Nacional de Enfermedades Crónicas
}

\author{
Carlos A A guilar-Salinas, MC, (1) Rosalba Rojas, MC, (2) Francisco J Gómez-Pérez, MC, ${ }^{(1)}$ \\ Victoria Valles, M en $C,{ }^{(1)}$ Aurora Franco, Lic, ${ }^{(2)}$ G ustavo $O$ laiz, $M$ en $C,{ }^{(2)}$ Roberto Tapia-Conyer, $M$ en $C, M P H,{ }^{(2)}$ \\ Jaime Sepúlveda, D r en C, ${ }^{(2)}$ Juan A Rull, MC. ${ }^{(1)}$
}

\begin{abstract}
Aguilar-Salinas CA, Rojas R, Gómez-Pérez FJ, VallesV,
Franco A, Olaiz G, Tapia-Conyer R, Sepúlveda J, Rull JA. Características de los casos con dislipidemias mixtas en un estudio de población: resultados de la Encuesta Nacional de Enfermedades Crónicas. Salud Publica Mex 2002;44:546-553.

El texto completo en inglés de este artículo está disponible en: http://www.insp.mx/salud/index.html
\end{abstract}

\section{Resumen}

Objetivo. Describir las características de los pacientes con hiperlipidemia mixta de acuerdo con los datos derivados de la Encuesta $\mathrm{N}$ acional de Enfermedades C rónicas. Material y métodos. Se realizó una entrevista en 1993, en 417 ciudades del país, y se midieron las concentraciones sanguíneas de lípidos séricos, glucosa e insulina en 2206 casos en un estudio poblacional. La diferencia entre los pacientes con dislipidemias mixtas y el resto de la población se estableció utilizando análisis de varianza o la prueba de ji cuadrada. Resultados. La hiperlipidemia mixta se encontró en 282 casos (12.8\%). Los individuos afectados tenían $42.7 \pm 12.6$ años. El $56 \%$ eran hombres; $46.4 \%$ tenían un colesterol HDL $<0.9 \mathrm{mmol} / \mathrm{l}$. La presencia de otros factores de riesgo fue común. La prevalencia de las hiperlipidemias mixtas fue alta aun en adultos jóvenes. Por medio de un modelo de regresión logística fue posible identificar la obesidad, la edad, el género, la región del país en donde residía el sujeto, la presencia de diabetes 0 de hipertensión arterial y la concentración de insulina de ayuno $>21 \mathrm{mU} / \mathrm{ml}$ como anormalidades asociadas a las dislipidemias mixtas. Conclusiones. La dislipidemia mixta es muy frecuente en adultos mexicanos. Se observa predo minantemente en hom-

\author{
Aguilar-Salinas CA, Rojas R, Gómez-Pérez FJ, VallesV, \\ Franco A, Olaiz G, Tapia-Conyer R, Sepúlveda J, Rull JA. \\ Characteristics of mixed hyperlipidemia cases \\ in a population-based study: Results \\ from the Mexican National Survey of Chronic Diseases. \\ Salud Publica Mex 2002;44:546-553. \\ The English version of this paper \\ is available: http://www.insp.mx/salud/index.html
}

\begin{abstract}
A bstract
Objective. To describe the characteristics of mixed hyperlipidemia cases, using data derived from the Encuesta $\mathrm{N}$ acional de Enfermedades C rónicas (Mexican N ational Survey of C hronic D iseases, EN EC). Material and Methods. The EN EC was conducted in 1993, in 417 Mexican cities. Blood measurements of lipids, glucose, and insulin were obtained from 2206 cases. Differences between dyslipidemia patients and non cases were obtained using analysis of variance or the chi-squared test. Results. Mixed hyperlipidemia was diagnosed in 282 subjects (12.8\%). Cases were $42.7 \pm 12.6$ years old. Fifty six percent were males and $46.4 \%$ had HDL cholesterol levels $<0.9 \mathrm{mmol} / \mathrm{l}$. 0 ther cardiovascular risk factors were also present. The prevalence of mixed hyperlipidemia was high even among young adults. A logistic regression model showed that obesity, age, male gender, residence in some regions of Mexico, diabetes, arterial hypertension, and fasting insulin levels $>21 \mathrm{mU} / \mathrm{ml}$, were factors associated with mixed hyperlipidemia. Conclusions. Mixed hyperlipidemia is a ver y common condition in Mexican adults. It is more common in males older than 30 years, with additional cardiovascular risk factors. Study findings suggest that the metabolic syndrome plays a role in the pathogene-
\end{abstract}

(1) Departamento de Endocrinología y Metabolismo del Instituto N acional de Ciencias Médicas y N utrición «Salvador Zubirán», México, D.F., México.

(2) Instituto Nacional de Salud Pública, Cuernavaca, Morelos, México.

Fecha de recibido: 25 de enero de 2002 - Fecha de aprobado: 7 de julio de 2002

Solicitud de sobretiros: MD Carlos Alberto A guilar-Salinas.Vasco de Q uiroga 15, 14000 México D.F., México. Correo electrónico:caguilarsalinas@ yahoo.com 
bres mayores de 30 años y los casos tienen otros factores de riesgo cardiovascular. Los datos sugieren que el síndrome metabólico participa en la génesis de la dislipidemia mixta. El texto completo en inglés de este artículo está disponible en: http://www.insp.mx/salud/index.html

Palabras clave: hiperlipoproteinemia tipo V; triglicéridos; síndrome metabólico; diabetes mellitus; hipertensión; México sis of this disorder.The English version of this paper is available: http://www.insp.mx/salud/index.html

Key words: hyperlipoproteinemia typeV; triglicerides; metabolic syndrome; diabetes mellitus; hypertension; Mexico
D urante las últimas tres décadas se ha puesto en duda el valor de los triglicéridos como factor de riesgo cardiovascular. ${ }^{1,2}$ Problemas metodológicos han sido una de las razones que originaron la controversia. La mayoría de los estudios epidemiológicos que han medido el riesgo cardiovascular al que se asocian las concentraciones de colesterol y triglicéridos lo han hecho asumiendo que los lípidos son variables independientes entre sí y que los padecimientos, en que estos parámetros se elevan, tienen una aterogenicidad similar. ${ }^{3}$ Sin embargo, estos supuestos no concuerdan con evidencias recientes. ${ }^{4}$ La concentración de estos lípidos reflejan el tipo y la cantidad de diversas clases de lipoproteínas con capacidades distintas para depositarse en una placa de ateroma. ${ }^{5}$ Así, una concentración muy elevada de triglicéridos ( $>500 \mathrm{mg} / \mathrm{dl},>5.6 \mathrm{mmol} /$ 1) no necesariamente es un marcador de riesgo cardiovascular, ya que esta anormalidad puede deberse a acumulación en el plasma de partículas aterogénicas (como las lipoproteínas de densidad intermedia) o lipoproteínas que por su tamaño no pueden depositarse en las placas de ateroma (como los quilomicrones). ${ }^{6} \mathrm{El}$ estudio the Prospective Cardiovascular Münster Study (PROCAM) analizó en forma prospectiva el riesgo asociado con diversas formas clínicas de dislipidemias. ${ }^{7}$ Sus datos demostraron que el riesgo cardiovascular de los pacientes con hipertrigliceridemia es variable y no puede ser analizado sin tomar en cuenta el colesterol total. Los casos con triglicéridos $\geq 200 \mathrm{mg} / \mathrm{dl}$ ( $2.24 \mathrm{mmol} /$ 1) y colesterol $<200 \mathrm{mg} / \mathrm{dl}$ ( $5.2 \mathrm{mmol} / \mathrm{l})$ tenían el mismo riesgo cardiovascular que los sujetos control. Por el contrario, los pacientes con dislipidemias mixtas (colesterol $\geq 5.2 \mathrm{mmol} / 1$ más triglicéridos $\geq 2.24 \mathrm{mmol} / \mathrm{l}$ ) tuvieron una incidencia de eventos cardiovasculares a seis años de 179 por 1000 casos, la cual es 13.8 veces mayor que la de los sujetos con concentraciones normales de lípidos. El riesgo asociado con este último grupo sólo fue menor al de los casos con las dislipidemias más severas (303 por 1000 casos). El riesgo es mayor cuando coexisten concentraciones bajas de colesterol-HDL. Aún más, el riesgo al que se asocia una concentración anormalmente alta de colesterol es dos o más veces mayor cuando coexiste una cifra de triglicéridos $>2.24 \mathrm{mmol} / \mathrm{l}$. Estos datos demuestran el aumento del riesgo cardiovascular asociado a las dislipidemias mixtas. Además, demuestran que la hipertrigliceridemia no puede ser analizada como una entidad única y que es necesaria la descripción por separado de los casos con y sin dislipidemia mixta.

Los mecanismos por los que se explica el mayor riesgo cardiovascular de las hiperlipidemias mixtas son múltiples. ${ }^{8}$ La existencia de una hiperlipidemia mixta es sinónimo del acúmulo en el plasma de uno o más tipos de lipoproteínas que tienen la capacidad de depositarse en las placas de ateroma. La mayoría de los casos tienen concentraciones plasmáticas anormalmente altas de remanentes de quilomicrones, de lipoproteínas de densidad intermedia o de lipoproteínas de baja densidad. ${ }^{9}$ Concentraciones altas de cualquiera de estas partículas tienen un efecto tóxico sobre las células endoteliales, aumentando su expresión de las moléculas de adhesión (VCAM-1, ICAM-1). Este evento facilita la adhesión de macrófagos y células mononucleares al endotelio y es el paso inicial para su migración al espacio subendotelial. ${ }^{10}$ $\mathrm{Al}$ incubar macrófagos con estas lipoproteínas se induce acumulación de ésteres de colesterol en su interior provocando su transformación en células espumosas. De mayor importancia es la evidencia aportada por los estudios en animales transgénicos en los que la mutación de genes involucrados en el catabolismo de estas partículas permite inducir ateroesclerosis severa y prematura. ${ }^{11,12}$ Además, en los pacientes con hiperlipidemias mixtas se han demostrado cambios protrombóticos y disminución de la actividad fibrinolítica, que se inducen durante la hidrólisis de las lipoproteínas ricas en triglicéridos. ${ }^{13,14}$ Por lo anterior, la hiperlipidemia mixta es una dislipidemia cuyo estudio y tratamiento permitirá prevenir un número significativo de eventos coronarios.

La prevalencia de las dislipidemias en México ha sido descrita en estudios pequeños que incluyeron predominantemente pacientes tratados en hospitales y en los de dos estudios de población con cobertura nacional. ${ }^{15-17}$ De estos últimos, sólo la Encuesta 
Nacional de Enfermedades Crónicas (ENEC) incluyó la medición de la concentración de triglicéridos. ${ }^{17}$ Los datos de la Encuesta muestran que la hipertrigliceridemia es una de las dislipidemias más frecuentes en población mexicana. En la población adulta urbana de entre 20 a 69 años, $24.3 \%$ tiene concentraciones de triglicéridos $\geq 2.24 \mathrm{mmol} / \mathrm{l}$. La prevalencia de hipertrigliceridemia en nuestra población es significativamente mayor a la descrita en otros grupos étnicos. ${ }^{18}$ Sin embargo, sólo 35\% de ellos (8.4\% de la población total) corresponden a hiperlipidemias mixtas. Este es el segmento de la población con hipertrigliceridemia que tiene el mayor riesgo cardiovascular. El objetivo de este informe es describir las características clínicas de los sujetos con hiperlipidemia mixta, incluyendo los padecimientos más frecuentemente asociados. Al ser las hiperlipidemias mixtas un síndrome, dicha descripción facilitará a los clínicos el abordaje diagnóstico de estos casos. El estudio se realizó en una población libre de sesgos de selección, pues se basó en datos derivados de una encuesta nacional en la que se utilizó un muestreo polietápico y aleatorio.

\section{Material y métodos}

Este es un estudio comparativo, transversal, que incluye individuos que viven en ciudades con más de 2500 habitantes ( $n=417$ ciudades). Se empleó un diseño probabilístico para el muestreo. Los detalles de la metodología fueron descritos en una publicación previa. ${ }^{17}$ El país fue dividido en cuatro regiones (norte, centro y sur) constituidas por $10 \mathrm{u} 11$ estados cada una. La cuarta zona correspondió al área metropolitana de la Ciudad de México. Una tabla de muestreo se diseñó con base en unidades geográficas definidas, para cada estado, por el Instituto Nacional de Geografía, Estadística e Informática. Se seleccionaron bloques de manzanas y viviendas en forma aleatoria, incluyendo en el estudio a todos los adultos de entre 20 a 69 años. Se excluyeron las instituciones militares y religiosas, hospitales o sitios que no fuesen utilizados como casa-habitación. La muestra fue representativa de la población urbana adulta que vive en México, la cual constituía en 1990, 71\% de la población total del país. Se incluyeron 4731 sujetos de 2030 domicilios por región, un promedio de 2.33 adultos por domicilio. Todos los hogares fueron visitados previa cita. El 15\% de ellos fue seleccionado al azar para ser visitado al inicio de la jornada. A ellos se les pidió que tuvieran un ayuno de 9 a 12 horas antes de la obtención de las muestras. En el resto de los casos la visita fue realizada durante la mañana. Con este diseño se puede medir la prevalencia de factores de riesgo y padecimientos que ocurren en más de $4 \%$ de la po- blación, con un error de estimación de 0.289 , y con una falta de respuesta de $30 \%$. Se obtuvo información de 15607 sujetos; la tasa de respuesta fue $82.5 \%$. El estudio se hizo siguiendo las normas de la declaración de Helsinki para estudios en humanos.

Se realizó una entrevista estructurada en todos los casos. Para ello se empleó un cuestionario previamente validado, cuyo objetivo fue obtener información demográfica, socioeconómica y sobre la historia médica personal y familiar. En la misma visita se midió la presión arterial, la talla y el peso. Para la toma de la presión arterial, se registraron la presión sistólica y la diastólica empleando un esfigmomanómetro de mercurio; los sujetos permanecieron sentados y en reposo por cinco minutos antes de la medición. Para la medición del peso y la talla, los pacientes se retiraron los zapatos y suéteres. El peso y la talla fueron obtenidos en una báscula con escalímetro, calibrada en cada hogar. El índice de masa corporal (IMC) se calculó dividiendo el peso $(\mathrm{Kg})$ entre la talla (expresada en metros al cuadrado). Los equipos fueron calibrados regularmente utilizando los materiales de referencia recomendados por los proveedores.

Se obtuvieron muestras sanguíneas en $77.6 \%$ de la población ( $n=14682$ ). Este informe incluye los resultados de 2206 casos en quienes se tomó la muestra después de 9 a 12 horas de ayuno, lo cual se requiere para evaluar con certeza el perfil de lípidos (13.9\% de la población total). ${ }^{19}$ Estos casos se distribuyeron en forma aleatoria entre la población; sin sesgos por región o estado socioeconómico. La toma de las muestras fue estandarizada durante un curso de entrenamiento de 28 semanas. Las muestras fueron obtenidas en los domicilios de los participantes. Antes de la obtención, los sujetos permanecieron sentados en reposo durante cinco minutos. Todas las mediciones de laboratorio fueron realizadas en el Departamento de Endocrinología y Metabolismo del Instituto Nacional de Ciencias Médicas y Nutrición "Salvador Zubirán".

La glucemia plasmática fue medida empleando el método de glucosa-oxidasa (Boehringer Mannheim). En la determinación del colesterol y de los triglicéridos se emplearon métodos enzimáticos comerciales (Boehringer Mannheim). El colesterol HDL fue medido después de precipitar las lipoproteínas que contienen la apoproteína B con fosfotungstato (Boehringer Mannheim). El coeficiente de variación intraensayo para el colesterol, los triglicéridos y el colesterol HDL fue de $3 \%, 5 \%$ y $5 \%$, respectivamente. La insulina fue medida con un método de ELISA con un sistema ES-33 (Boehringer Mannheim). Los ensayos se realizaron de acuerdo a las instrucciones de los fabricantes, con los calibradores y antisueros adecuados. Todas las muestras 
permanecieron congeladas a $-80^{\circ} \mathrm{C}$ hasta su análisis; el periodo máximo de almacenamiento fue de 12 meses. Nuestro laboratorio sigue los procedimientos de estandarización recomendados por la Organización Mundial de la Salud, incluyendo el uso de calibradores externos.

Los casos con triglicéridos $\geq 200 \mathrm{mg} / \mathrm{dl}(2.24$ $\mathrm{mmol} / \mathrm{l}$ ) y colesterol $\geq 200 \mathrm{mg} / \mathrm{dl}$ (5.2 mmol/l) fueron considerados como afectados por la hiperlipidemia mixta. ${ }^{20}$ Los mismos puntos de corte se emplearon para el diagnóstico de la hipertrigliceridemia y de la hipercolesterolemia, respectivamente. Los casos con triglicéridos $<200 \mathrm{mg} / \mathrm{dl}(2.24 \mathrm{mmol} / \mathrm{l})$ y colesterol $<200$ $\mathrm{mg} / \mathrm{dl}(5.2 \mathrm{mmol} / \mathrm{l})$ fueron clasificados como normolipidémicos. El colesterol noHDL fue obtenido de la resta de la concentración del colesterol-HDL del colesterol sanguíneo. ${ }^{21}$ Sobrepeso fue definido como un IMC entre 25 y $30 \mathrm{~kg} / \mathrm{m}^{2}$. Se consideró a un sujeto con obesidad si tenía un IMC $\geq 30 \mathrm{~kg} / \mathrm{m}^{2}$. La presencia de diabetes mellitus se estableció por el diagnóstico previo de la enfermedad o glucemia de ayuno $\geq 7 \mathrm{mmol} / \mathrm{l}$. La hipertensión arterial se diagnosticó si la presión sistólica fue $\geq 140 \mathrm{~mm} \mathrm{Hg}$ o la presión diastólica fue $\geq 90 \mathrm{~mm} \mathrm{Hg}$, o si la persona utilizaba algún antihipertensivo. La presión arterial fue medida en dos ocasiones si en la visita, la presión fue $\geq 120 / 80$. La cardiopatía isquémica se diagnosticó cuando el entrevistado refirió haber sufrido un infarto del miocardio. El modelo de homeostasis (HOMA-IR) ${ }^{22}$ se utilizó para detectar la presencia de resistencia a la insulina; un valor mayor de 2.4 fue considerado como diagnóstico. Este punto de corte corresponde a la percentila 90 de la población estudiada. Puntos de corte similares han sido empleados en otros reportes. ${ }^{23}$ El consumo de tabaco se consideró positivo si el individuo refirió haber fumado durante el mes previo al estudio. El climaterio fue definido en mujeres a las que un médico previamente las hubiese diagnosticado, o que no hubiesen tenido una menstruación durante el año anterior.

La base de datos fue validada, corrigiendo información faltante e inconsistente. El análisis descriptivo se realizó empleando promedios o medianas y desviaciones estándar para las variables continuas. La prueba de ANOVA se utilizó para comparar diferencias entre los subgrupos de la población. Las variables categóricas se compararon con la prueba de ji cuadrada y la corrección de Yates o la prueba exacta de Fisher cuando fue necesario; se obtuvieron razones de momios para estimar el riesgo y sus intervalos de confianza. Se generó un modelo de regresión logística para identificar las anormalidades asociadas con la hiperlipidemia mixta. En él se incluyó a la hiperlipidemia mixta como variable dependiente y al resto de los parámetros que tuviesen una prueba de ji cuadrada mayor de 0.1 . Los análisis estadísticos se realizaron con el programa SAS 8.1 y SPSS para Windows.

\section{Resultados}

De los 2206 individuos con muestras de ayuno (929 hombres y 1277 mujeres), 282 (12.8\%) tuvieron hiperlipidemia mixta. En el cuadro I, se muestran las características de los casos afectados y su comparación con la población normolipidémica (colesterol $<5.2$ $\mathrm{mmol} / \mathrm{l}$ y triglicéridos $<2.24 \mathrm{mmol} / \mathrm{l}$ ). Los casos con hiperlipidemia mixta fueron sujetos con un promedio de edad de $42.7 \pm 12.6$ años. El $56 \%$ fueron hombres. La concentración promedio de colesterol del grupo afectado fue de $6.18 \pm 0.73 \mathrm{mmol} / \mathrm{l}$, la de triglicéridos

\section{Cuadro I Características de los pacientes CON HIPERLIPIDEMIAS MIXTAS Y SU COMPARACIÓN con el resto de la población. Encuesta Nacional de Enfermedades Crónicas. México, 1993}

\begin{tabular}{|c|c|c|c|}
\hline & $\begin{array}{l}\text { Hiperlipidemia } \\
\quad(n=282)\end{array}$ & $\begin{array}{l}\text { Resto de la } \\
\text { población* } \\
\text { (n=1 924) }\end{array}$ & $\begin{array}{c}\text { Sujetos } \\
\text { normolipidémicos } \\
(n=1356)\end{array}$ \\
\hline dad (años) & $42.7 \pm 12.6^{\ddagger}$ & $34.9 \pm 12.5^{\S}$ & $32.5 \pm 11$ \\
\hline Sexo masculino $(n(\%))$ & $158(56.3)^{\ddagger}$ & $771(40.07)$ & $512(31.2)$ \\
\hline Indice de masa corporal $\left(\mathrm{kg} / \mathrm{m}^{2}\right)$ & $28.5 \pm 4.7^{\ddagger}$ & $26.2 \pm 5.2$ & $25.5 \pm 5.1$ \\
\hline Presión sistólica (mmH g) & $134 \pm 18+$ & $126 \pm 17$ & $121 \pm 15$ \\
\hline Presión diastólica (mmHg) & $89 \pm 14^{\ddagger}$ & $82 \pm 12$ & $78 \pm 10$ \\
\hline Colesterol (mmol/l) & $6.18 \pm 0.73^{\ddagger}$ & $4.47 \pm 0.86$ & $4.2 \pm 0.5$ \\
\hline Triglicéridos (mmol/l) & $3.93 \pm 2.2^{\ddagger}$ & $1.57 \pm 0.95^{\S}$ & $1.35 \pm 0.44$ \\
\hline Colesterol HDL (mmol/l) & $0.93 \pm 0.2^{\ddagger}$ & $1.02 \pm 0.2$ & $1.05 \pm 0.2$ \\
\hline Colesterol noHDL (mmol/l) & $5.23 \pm 0.7^{\ddagger}$ & $3.47 \pm 0.8$ & $3.13 \pm 0.5$ \\
\hline HOMA-IR & $8.04 \pm 6.5^{\ddagger}$ & $4.35 \pm 6.3$ & $2.8 \pm 4.8$ \\
\hline Glucemia (mmol/l) & $6.7 \pm 3.8^{\ddagger}$ & $5.3 \pm 2.1$ & $5.3 \pm 1.8$ \\
\hline Diabetes (\%) & $21.4^{\ddagger}$ & 6.8 & 2.8 \\
\hline $\mathrm{IMC}>30 \mathrm{~kg} / \mathrm{m}^{2}(\%)$ & $29.3^{\ddagger}$ & 20.4 & 17.2 \\
\hline Climaterio (\%) & $55.5^{\ddagger}$ & 28.7 & 21.4 \\
\hline Hipertensión arterial (\%) & $42.7^{\ddagger}$ & 23.4 & 13.5 \\
\hline \multicolumn{4}{|c|}{ 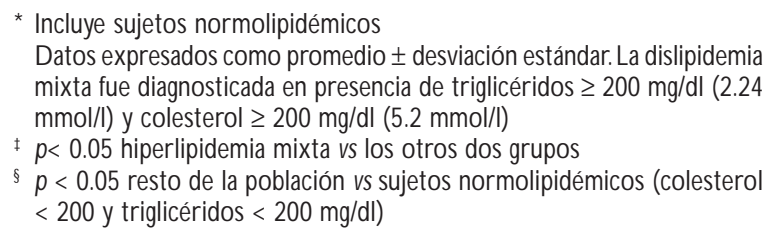 } \\
\hline
\end{tabular}

$I M C=$ Indice de masa corporal. HOMA = Homeostasis model assesment, indicador empleado para estimar resistencia a la insulina 
de $3.93 \pm 2.2 \mathrm{mmol} / \mathrm{l}$, y la de colesterol noHDL de 5.23 $\pm 0.76 \mathrm{mg} / \mathrm{dl}$. Todos estos valores fueron significativamente más altos que los observados en los sujetos sin hiperlipidemia mixta. En 131 de los sujetos afectados (46.4\%) el colesterol HDL fue menor de $0.9 \mathrm{mmol} / \mathrm{l}$. Esta anormalidad fue significativamente más frecuente en este grupo que en el resto de la población (27.4\%, $p$ $<0.001)$. La presencia de otros factores de riesgo fue común en estos casos. Consumían tabaco regularmente $57 \%$ de ellos. La diabetes mellitus fue diagnosticada en 60 casos (21.4\% de los casos con hiperlipidemia mixta). La hipertensión arterial se encontró en 119 casos $(42.6 \%)$. La frecuencia de estas comorbilidades fue significativamente menor en el resto de la población $(6.8 \%$ con diabetes y $23.5 \%$ con hipertensión arterial, $p<0.001$ para ambos fenómenos).

La prevalencia de las hiperlipidemias mixtas por edad y género se muestra en el cuadro II. Su frecuencia es alta aun en adultos jóvenes ( $5.1 \%$ de los sujetos entre 20 y 30 años de edad). La prevalencia en las mujeres es similar a la de los hombres, pero con un desfase de 10 años más en la edad. En los hombres se observa una disminución en la prevalencia en el grupo de mayor edad. Esta anormalidad fue más frecuente en la zona centro del país y en la Ciudad de México (14.7 y 15.2\% respectivamente) en comparación con las zonas norte y sur (10.4 y $10.9 \%$ respectivamente, $p<0.001)$.

Se buscaron condiciones con las cuales la hiperlipidemia mixta se encontrara asociada. Entre los pacientes con dislipidemias mixtas, $51.3 \%$ tenían sobrepeso y $30.7 \%$ eran obesos. Sólo 18\% de los casos tenían un IMC $<25 \mathrm{~kg} / \mathrm{m}^{2}$, porcentaje que fue significativamente menor al del resto de la población $(42.3 \%, p<0.001)$. La dislipidemia mixta fue más frecuente en el climaterio (53.3 vs $29.3 \%, p<0.001)$ y entre los fumadores (15 vs $10.4 \%, p=0.001)$. Su frecuencia no fue mayor en casos con creatinina sérica mayor de $2 \mathrm{mg} / \mathrm{dl}$. Un valor de HOMA mayor de 2.4 fue significativamente más frecuente en los individuos afectados que en el resto de la población $(79.8 \%$ vs $57.8 \%$, $p<0.01)$. En el cuadro III se observa que la hiperlipidemia mixta está asociada de manera independiente con la diabetes mellitus ( $R M$ 5.39, IC 95\% 3.6-7.87). Este valor permaneció significativo en un modelo que ajustó por edad, género e índice de masa corporal. Esta razón de momios fue la más alta de las incluidas en el análisis. La dislipidemia mixta se encontró asociada a la hipertensión arterial (RM 3.09, IC 95\% 2.35-4.06), aun al tomar en cuenta los confusores antes mencionados.

Los factores asociados con la presencia de hiperlipidemia mixta identificados con un modelo de regresión logística se muestran en el cuadro IV. El modelo tuvo un valor de $p<0.001$. Las variables inclui-

\section{Cuadro II \\ Porcentaje de la población afectado POR UNA HIPERLIPIDEMIA MIXTA. ENCUESTA NaCIONAL de Enfermedades Crónicas. México, 1993}

$$
\begin{array}{cc}
\begin{array}{c}
\text { Dislipidemias mixtas } \\
\mathrm{n}(\%)
\end{array} & \begin{array}{c}
\text { Porcentaje de los casos } \\
\text { con dislipidemias mixtas } \\
\text { con } \mathrm{HDL}<35 \mathrm{mg} / \mathrm{dl} \\
(0.9 \mathrm{mmol} / \mathrm{l}) \\
\mathrm{n}(\%)
\end{array}
\end{array}
$$

\begin{tabular}{|c|c|c|c|c|}
\hline Población total & & & & \\
\hline $20-29$ años ( $n=872)$ & 45 & (5.1) & 26 & (56.8) \\
\hline 30-39 años ( $n=582)$ & 84 & $(14.4)$ & 43 & $(50.6)$ \\
\hline $40-49$ años ( $n=374)$ & 65 & $(17.0)$ & 28 & (43.7) \\
\hline $50-59$ años $(n=238)$ & 58 & $(24.2)$ & 27 & $(47.4)$ \\
\hline $60-69$ años ( $n=140)$ & 30 & $(21.5)$ & 8 & $(25.8)$ \\
\hline Total $(n=2206)$ & 282 & $(12.8)$ & 132 & $(46.6)$ \\
\hline
\end{tabular}

Hombres

\begin{tabular}{lrrrr}
\hline 20-29 años $(n=380)$ & 31 & $(8.2)$ & 21 & $(67.7)$ \\
\hline 30-39 años $(n=234)$ & 55 & $(23.6)$ & 38 & $(69.1)$ \\
\hline 40-49 años $(n=158)$ & 32 & $(20.2)$ & 22 & $(68.7)$ \\
\hline 50-59 años $(n=96)$ & 28 & $(28.5)$ & 14 & $(50)$ \\
\hline 60-69 años $(n=61)$ & 12 & $(19.3)$ & 0 & $(0)$ \\
\hline Total $(n=929)$ & 158 & $(17.0)$ & 95 & $(60.1)$
\end{tabular}

Mujeres

\begin{tabular}{lrrrl}
\hline 20-29 años $(n=487)$ & 13 & $(2.6)$ & 4 & $(30.7)$ \\
\hline 30-39 años $(n=347)$ & 28 & $(8.2)$ & 4 & $(14.2)$ \\
\hline $40-49$ años $(n=217)$ & 32 & $(14.7)$ & 6 & $(18.7)$ \\
\hline 50-59 años $(n=145)$ & 31 & $(21.4)$ & 14 & $(45.1)$ \\
\hline $60-69$ años $(n=81)$ & 19 & $(23.1)$ & 8 & $(42.1)$ \\
\hline Total $(n=1277)$ & 123 & $(9.6)$ & 36 & $(29.2)$
\end{tabular}

La dislipidemia mixta fue diagnosticada en presencia de triglicéridos $\geq 200$ $\mathrm{mg} / \mathrm{dl}(2.24 \mathrm{mmol} / \mathrm{l})$ y colesterol $\geq 200 \mathrm{mg} / \mathrm{dl}(5.2 \mathrm{mmol} / \mathrm{l})$

das fueron la obesidad, la edad, el género, la región del país en que residía el sujeto, la presencia de diabetes o de hipertensión arterial y la concentración de insulina de ayuno $>21 \mathrm{mU} / \mathrm{ml}$. En el mismo cuadro se muestran los riesgos relativos para la presencia de hiperlipidemia mixta con los que se asocian dichas variables.

Finalmente, un valor de colesterol HDL menor de $0.9 \mathrm{mmol} / 1$ se asoció con diferencias en la concentración de lípidos en el grupo de pacientes con hiperlipidemias mixtas. Los casos con hipoalfalipoproteinemia tenían valores de colesterol noHDL (5.41 \pm 0.71 vs $5.1 \pm$ $0.71, p<0.001)$ y triglicéridos $(4.42 \pm 2.4$ vs $3.5 \pm 2.05$, $p<0.001$ ) significativamente mayores que el resto de los sujetos con hiperlipidemia mixta. Una proporción 


\section{Cuadro III \\ AsOCIACIÓN CON DIABETES O HIPERTENSIÓN ARTERIAL DE DIVERSAS FORMAS CLÍNICAS DE DISLIPIDEMIA.* Encuesta Nacional de Enfermedades Crónicas. MÉXICO, 1993

$\begin{array}{lcc}\text { Dislipidemia } & \begin{array}{c}\text { Diabetes mellitus } \\ \text { (RM IC 95\%) }\end{array} & \begin{array}{c}\text { Hipertensión arterial } \\ \text { (RM IC 95\%) }\end{array} \\ \begin{array}{l}\text { Hipercolesterolemia } \\ \text { (Colesterol }>5.2 \mathrm{mmol} / \mathrm{l}\end{array} & 2.15(1.59-2.91) & 2.16(1.76-2.65) \\ + \text { triglicéridos }<2.2 \mathrm{mmol} / \mathrm{l}) & & \end{array}$

Hipertrigliceridemia

$4.5(3.32-6.09) \quad 2.25(1.82-2.77)$

(Colesterol $<5.2 \mathrm{mmol} / \mathrm{l}$

+ triglicéridos $>2.2 \mathrm{mmol} / \mathrm{l})$

Hiperlipidemia mixta

$5.39(3.69-7.87) \quad 3.09(2.38-4.06)$

(Colesterol $>5.2 \mathrm{mmol} /$

+ triglicéridos $>2.2 \mathrm{mmol} / \mathrm{l})$

$1.22(0.99-1.5)$

Hiperlipidemia mixta más

colesterol HDL bajo

$3.39(2.09-5.52) \quad 2.64(1.83-3.82)$

Colesterol $>5.2 \mathrm{mmol} / \mathrm{l}$

+ triglicéridos $>2.2 \mathrm{mmol} / \mathrm{l}$

+ colesterol HDL $<0.9 \mathrm{mmol} / \mathrm{l})$

* Las razones de momios están ajustadas para la edad, sexo e índice de masa corporal

significativamente mayor de ellos eran hombres (72.5 vs $41.7 \%$, $p<0.001)$. Aún de mayor importancia es la distribución por edad de los casos en que coexistió una concentración baja de colesterol HDL (cuadro II). En los sujetos menores de 30 años, $56.8 \%$ de los casos con hiperlipidemia mixta tuvieron el colesterol HDL bajo. Este porcentaje se mantuvo cercano a $50 \%$ en los sujetos entre 30 y 59 años y en el grupo de mayor edad (60-69 años) se redujo a $25.8 \%(p=0.01)$. Este fenómeno se observó predominantemente en los hombres. Estos datos sugieren que el riesgo cardiovascular es probablemente mayor en los casos en que coexisten ambas anormalidades y que la combinación de estos defectos se observa sobre todo en los grupos de menor edad.

\section{Discusión}

Las dislipidemias mixtas son un grupo de alteraciones del metabolismo de lipoproteínas que se asocian con un riesgo cardiovascular alto. Su aterogenicidad quedó

\section{Cuadro IV \\ Factores asociados CON LA PRESENCIA DE LA HIPERLIPIDEMIA MIXTA ESTIMADA POR MEDIO DE UN MOdelo de REgResión logística. EnCUeSta NaCional de Enfermedades Crónicas. México, 1993}

$\begin{array}{lcccc}\text { Parámetro } & \text { Estimado } & \begin{array}{c}\text { Error } \\ \text { estándar }\end{array} & p & \text { RM (IC 95\%) } \\ \begin{array}{lcccc}\text { Intercepto } & -4.5029 & 0.4338 & <0.001 & \\ \hline \begin{array}{l}\text { Indice de masa } \\ \text { corporal }>30 \mathrm{~kg} / \mathrm{m}^{2}\end{array} & 0.02 & 0.004 & <0.001 & 1.02(1.01-1.03) \\ \hline \text { Género masculino } & 0.241 & 0.0 .07 & <0.001 & 1.62(1.22-2.14) \\ \hline \begin{array}{l}\text { Residencia en la } \\ \text { Ciudad de México }\end{array} & 0.312 & 0.118 & 0.008 & 1.68(1.16-2.44) \\ \hline \begin{array}{l}\text { Residencia en la } \\ \text { Región norte }\end{array} & -0.332 & 0.124 & 0.007 & 0.78(0.63-0.96) \\ \hline \text { Insulina }>21 \mathrm{mU} / \mathrm{ml} & 0.011 & 0.002 & <0.001 & 1.01(1.006-1.017) \\ \hline \text { Diabetes } & 0.326 & 0.098 & <0.001 & 1.92(1.306-2.823) \\ \hline \text { Hipertensión arterial } & 0.209 & 0.074 & <0.001 & 1.52(1.136-2.037)\end{array}\end{array}$

Valor de $p$ del modelo $<0.00$

establecida por la elevada tasa de eventos cardiovasculares que se observó en el estudio PROCAM. En dicho estudio, los pacientes con hiperlipidemia mixta tuvieron una tasa de eventos coronarios sólo menor al de los casos con las dislipidemias más severas. El beneficio del tratamiento hipolipemiante en pacientes con hiperlipidemia mixta es aún mayor que el observado en casos con hipercolesterolemia. ${ }^{24}$ Pese a ello, existen pocos estudios en población general que hayan establecido la prevalencia de la hiperlipidemia mixta. La mayoría de los estudios han investigado la prevalencia de hipercolesterolemia y de hipertrigliceridemia como si fuesen variables independientes, cuando, en realidad, la concentración de los lípidos séricos es indicadora del acúmulo en el plasma de una o más clases de lipoproteínas aterogénicas. Por otra parte, la mayoría de los estudios han sido realizados en poblaciones atendidas en hospitales o afectadas por cardiopatía isquémica, características que limitan las conclusiones y que impiden su extrapolación a la población general. ${ }^{25-27}$ Nuestros datos demuestran que la hiperlipidemia mixta es una dislipidemia muy frecuente en los adultos mexicanos que viven en zonas urbanas. La prevalencia observada es aún mayor que la descrita en el estudio PROCAM, en el cual se incluyeron 17426 hombres y 8061 mujeres alemanas. Como en el estudio PROCAM, las dislipidemias mixtas fueron más frecuentes en los hombres y su prevalencia 
aumentó con la edad. En ambas poblaciones, la prevalencia de esta dislipidemia es casi cuatro veces más alta en los hombres jóvenes ( $<30$ años) que en las mujeres de la misma edad. En contraste con la población alemana, en la que la prevalencia en los hombres fue tres o cuatro veces mayor a la de las mujeres sin importar el grupo de edad, en la población mexicana la prevalencia en las mujeres fue similar a la de los hombres 10 años más jóvenes. La diferencia en la prevalencia de la dislipidemia mixta sugiere que factores genéticos o ambientales contribuyen de manera diferenciada en la presentación de las dislipidemias mixtas y que estos factores deben ser distintos en las poblaciones caucásica y mexicana.

En los casos con hiperlipidemias mixtas se observó una asociación con otros factores de riesgo cardiovascular. Comparados con el resto de la población, los individuos afectados tuvieron un riesgo relativo de 5.3 (IC 95\% 3.6-7.9) para diabetes y de 3.1 (IC 95\% 2.3-4.1) para hipertensión arterial. Estos riesgos son independientes de la edad, índice de masa corporal y género. Además, los pacientes con hiperlipidemias mixtas tuvieron colesterol HDL menor y colesterol no HDL mayor que el resto de los sujetos. Aún más, un alto porcentaje de ellos tuvo sobrepeso u obesidad. Paradójicamente, en estos casos se fumaba o se había fumado con mayor frecuencia que en el resto de la población. Estos datos demuestran que la búsqueda intencionada de los factores de riesgo cardiovascular debe ser una práctica rutinaria en la evaluación de los pacientes con hiperlipidemias mixtas.

La coexistencia con colesterol HDL bajo potencia a la aterogenicidad de la dislipidemia mixta. Los datos del estudio PROCAM y del informe reciente de los estudios de Caerphilly y Speedwell ${ }^{28}$ muestran que la tasa de eventos cardiovasculares aumenta al menos dos veces al coexistir ambas anormalidades. En los casos con hipoalfalipoproteinemia e hiperlipidemia mixta reportados se observó una concentración mayor de colesterol no HDL y de triglicéridos que en los casos restantes con hiperlipidemia mixta. Estas anormalidades adicionales pueden ser una de las explicaciones de la mayor aterogenicidad observada. Una evidencia indirecta de la aterogenicidad de este perfil de lípidos se encuentra en la distribución por edades de los casos afectados. En los sujetos de sexo masculino con hiperlipidemia mixta se observó concordancia de la frecuencia máxima de estas anormalidades con el periodo de la vida en el que ocurre el mayor número de eventos vasculares. La mayor letalidad de la combinación hiperlipidemia mixta / colesterol HDL bajo se demuestra en la ausencia de sujetos mayores de 60 años que presentan esta anormalidad.
Por medio de un modelo de regresión logística fue posible identificar algunos factores causales putativos de esta dislipidemia en población mexicana. Estos incluyen la obesidad, la diabetes, las concentraciones elevadas de insulina en suero, el género masculino y el lugar de residencia. Una limitante de este análisis es la falta de una evaluación detallada de los antecedentes heredofamiliares y la ausencia de un registro de la alimentación y de la actividad física. Por ello, no se pueden evaluar con certeza en este informe los componentes genéticos y nutrimentales, ni la participación de algunas hiperlipidemias primarias como la hiperlipidemia familiar combinada y la disbetalipoproteinemia. ${ }^{29}$ Sin embargo, los datos sugieren que el síndrome metabólico es una causa frecuente de dislipidemia mixta en México. La afirmación se basa en la asociación de las concentraciones altas de insulina con esta anormalidad y en la mayor frecuencia de valores anormalmente altos de HOMA-IR (un indicador de resistencia a la insulina) encontrada en los casos con esta dislipidemia. La participación del síndrome metabólico puede ser la explicación para la elevada frecuencia de esta dislipidemia en nuestra población. ${ }^{30}$ Estos datos sugieren que hiperlipidemias primarias asociadas con el síndrome metabólico, como la hiperlipidemia familiar combinada, ${ }^{31}$ podrían estar presentes en una proporción significativa de los casos. Finalmente, esta observación, además, permite suponer que programas que reduzcan la prevalencia de obesidad (en especial en los hombres) serán eficaces para reducir la frecuencia de la dislipidemia mixta.

En conclusión, la dislipidemia mixta es muy frecuente en población urbana adulta mexicana, se observa más en hombres mayores de 30 años y se asocia, con frecuencia, con otros factores de riesgo cardiovascular. Los resultados sugieren que el síndrome metabólico juega un papel en la génesis de la dislipidemia mixta. Por lo anterior, se refuerza la necesidad de desarrollar y aplicar programas preventivos que disminuyan la prevalencia de obesidad y diabetes, ya que como beneficio adicional tendrán un impacto positivo en el número de pacientes afectados por una de las formas de dislipidemia más aterogénica y mórbida.

\section{Referencias}

1. Austin M, Hokanson JE, Edwards K. Hypertriglyceridemia as a cardiovascular risk factor. Am J Cardiol 1998;81(4A): 7B-12B.

2. Kesäniemi YA. Serum triglycerides and clinical benefit in lipid lowering trials. Am J Cardiol 1998;81(4A): 70B-73B.

3. Sundquist J,W inkleby MA, Pudaric S. C ardiovascular disease risk factors among older black, Mexican-American, and white women and men: An 
analysis of NHANES 1988-1994. Third National Health and Nutrition Examination Survey. J Am Geriatr Soc 2001;49:109-116.

4. Gaw A. Evidence based approach for the management of mixed hyperlipidemia.A therosclerosis 1998;137 Suppl:S97-S100.

5. Santamarina-Fojo S.The familial chylomicronemia syndrome. Endocrinol Metab C lin N orth Am 1998; 27:551-567.

6. Gómez-Pérez FJ, Aguilar-Salinas C A. Hiperlipoproteinemias primarias. En: Posadas C, ed. Dislipidemias y ateroesclerosis. México, D.F.: Editorial Interamericana: McG raw-Hill, 1995: 87-104.

7. Assmannn G, Schulte H. Results and conclusions of the Prospective Cardiovascular Münster (PRO CAM) Study. En: Assmann G, ed. Lipid Metabolism D isorders and Coronary Heart D isease. MMV Medizin Verlag 1993: 21-67.

8. Frost P, Havel R. Rationale for use of non high density lipoprotein cholesterol rather than low density lipoprotein cholesterol as a tool for lipoprotein cholesterol screening and assessment of risk and therapy.Am J Cardiol 1998;81(4A): 26B-31B.

9. Sniderman AD, Vu H, Cianflone K. The effect of moderate hypertriglyceridemia on the relation of plasma total and LDL apoB levels. Atherosclerosis 1991; 89:109-116.

10. Jiang $F$, Gibson AP, Dusting GJ. Endothelial dysfunction induced by oxidized low-density lipoproteins in isolated mouse aorta: A comparison with apolipoprotein -E deficient mice. Eur J Pharmacol 2001; 424 :141149 .

11. Jong MC, H avekes LM. Insights into apolipoprotein $C$ metabolism from transgenic and gene targeted mice. Int J Tissue React 2000;22:59-66.

12. McG illicuddy CJ,C arrier MJ,W einberg PD. D istribution of lipid deposits around aortic branches of mice lacking $L D L$ receptors and apolipo protein E.Arterios Thromb Vasc Biol 2001;21:1220-1225.

13. C hadarevian R, Brucker E, D ejager S, Presberg P,Turpin G . Relationship between triglycerides and factor VIIc and PAI-1: Lack of threshold value. Thromb Res 1999:96:175-182.

14. Li XN , G renett HE, Benza RL, D emissie S, Brown SL, Tabengwa EM et al. Genotype-specific transcriptional regulation of PAI-1 expression by hypertriglyceridemic VLDL and $L p(a)$ in cultured human endothelial cells. Arterios Thromb Vasc Biol 1997;17:3215-3223.

15. Fanghanel-Salmón G, Sánchez-Reyes L,A rellano S, Valdés S, Chavira J, Rascón A. The prevalence of risk factors for coronary disease in workers of the Hospital General de Mexico. Salud Publica Mex 1997; 39:427-432. 16. Posadas-Romero C, Tapia-C onyer R, Lerman-Garber I, ZamoraGonzález J, C ardoso-Saldaña G, Salvatierra-Izaba B et al.C holesterol levels and prevalence of hypercholesterolemia in a Mexican adult population. Atherosclerosis 1995;118:275-284.

17. A guilar-Salinas CA, 0 laiz G,Valles V, Ríos JM, Gómez-Pérez FJ, Rull JA et al. High prevalence of low HDL cholesterol concentrations and mixed hyperlipidemia in a Mexican nation wide survey. J Lipid Research 2001; 42:1298-1307.

18. Bhopal R, Unwin N,W hite M, Yallop J,W alker L,Alberti KGGM et al. $\mathrm{H}$ eterogeneity of coronary heart disease risk factors in Indian, Pakistani,
Bangladeshi, and European origin populations: C ross sectional study. BMJ 1999; 319: 215-220.

19. Expert panel on detection, evaluation and treatment of high blood cholesterol in adults. Executive summary of the third report of the $\mathrm{N}$ ational Cholesterol Education Program (N CEP) Expert panel on detection, evaluation and treatment of high cholesterol.JAMA 2001;285:2486-2497. 20. Ballantyne C, G rundy SM , O berman A, Kreisberg R, H avel R, Frost P et al. Hyperlipidemia: Diagnostic and therapeutic perspectives. J Clin Endoc Metab 2000; 85:2089-2112

21. Havel RJ, Frost P.The role of non-high-density lipoprotein cholesterol in evaluation and treatment of lipid disorders. J Clin Endocrinol Metab 2001; 85:2105-2108.

22. Matthews D, H osler JP, Rudenski AS, N aylor BA, Treacher DF,Turner $R$. Homeostasis model assessment:Insulin resistance and B-cell function from fasting plasma glucose and insulin concentration in man. Diabetologia 1985;28:412-419.

23.Taniguchi A, Fukushima M, Sakai M, Kataoka K, N agata I, D oi K et al.The role of body mass index and triglycerides levels in identifying insulinsensitive and insulin-resistant variants in Japanese non-insulin dependent diabetic patients. Metabolism 2000; 49: 1001-1005.

24. Ballantyne C, 0 Isson AG, C ook TJ, Mercuri M, Pedersen T, Kjekshus J. For the Scandinavian Simvastatin Survival Study (4S) Group. Influence of low high-density lipoprotein cholesterol and elevated triglycerides in coronary heart disease events and response to simvastatin therapy in 45 . Circulation 2001;104:3046-3051.

25. Ezenwaka CE, 0 ffiah N V.C ardiovascular risk in obese and nonobese patients with type 2 diabetes in the west indies.J Biomed Sci 2001;8:314320.

26. Rajmohan L, D eepa R, Mohan A, MohanV.Association between isolated hypercholesterolemia, isolated hypertriglyceridemia and coronary artery disease in so uth Indian type 2 diabetic patients. Indian Heart J 2000;52:400406.

27. Miller M. Triglyceride as a risk factor, epidemiology. Lipids 1999;34 Suppl:S267.

28. Yarnell JW G, Patterson CC, Sweetnam PM, Thomas HF, Bainton D, Elwood PC et al. Do total and high density lipoprotein cholesterol and triglycerides act independently in the prediction of ischemic heart disease? Ten-year follow- up of C aerphilly and Speedwell C ohorts.ArteriosThromb Vasc Biol 2001;21:1340-1345.

29. Grundy SM, Chait A, Brunzell J. Familial combined hyperlipidemia workshop. Arteriosclerosis 1987;7:203-207.

30.A guilar-Salinas C A,Vázquez-C hávez C, G amboa-Marrufo R, G arcía-Soto $\mathrm{N}$, Ríos-González JJ, Holguín R et al. Prevalence of obesity, diabetes, hypertension and tobacco consumption in an urban adult Mexican population. Arch Med Res 2001;32:446-453.

31. Eckel R. Familial combined hyper lipidemia and insulin resistance. D istant relatives linked by intra-abdominal fat? Circulation 2001;21:469-470. 